\title{
About an Unbiased Estimate of the Gradient with Minimum Variance in the Planning of the Experiment
}

\author{
Sidnyaev N.I.", Razgulyaev S.V., Shafikova S.E. \\ Fundamental Sciences Faculty, The Bauman Moscow State Technical University,105005,ul.Baumanskay 2-ya, 5, Moscow, Russia \\ *Corresponding Author: sidnyaev@bmstu.tu
}

Copyright (C) 2014 Horizon Research Publishing All rights reserved.

\begin{abstract}
This paper considers algorithms of search of an extremum, to solve the problem of planning the experiment using the gradient method. A feature of the algorithm is that when searching for the motion (when the maximum) does not occur in the direction of the gradient, which is unknown to us, but its estimate. Estimate of the gradient at the point when this factor space is based on the results of measurements carried out in the neighborhood. Researcher's task is to build a sensible plan with center to determine the estimate of the gradient in it.
\end{abstract}

Keywords Theory of Experiment Planning, Unbiased Estimate of the Gradient, The Response Function, The Method of Least Squares

\section{Introduction}

The task of finding the extremum of the mean characteristics, usually the task of finding the extremum of the response function $\eta=f\left(x_{1}, x_{2}, \ldots, x_{k}\right)$. Search extremum response function is performed by the response surface study. This study is carried out by measuring the response surface at different points in the factor space.

Use for this purpose immediately known methods of finding the extremum function of many variables cannot be as " measurement " of the response function at each point in the factor space, which put the experience happens to fail.

1. One of the most famous classes of gradient methods for searching the extremes of the response function in the practice of experimental plan can be considered a method developed by Box and Wilson [1, 2]. The idea behind it is to use the method of steep climbing (steepest ascent) in conjunction with the series planned factorial experiment to find the gradient estimation. When applying gradient methods for searching the extremes of the response function of one of the most important is the problem of statistical estimation of the gradient components [3-5]. Therefore, in describing the method of Box and Wilson this problem is considered the most complete. Research issues of statistical estimation of the gradient when searching, is of great importance for the understanding of the use of gradient methods for planning the experiment. In general, the method of Box and Wilson is to repeat the procedure:

- build - factorial experiment [3] in the neighborhood of a point;

- estimating the gradient - calculation at this point, the results of the experiment;

- finding estimates of the maximum (minimum) of the response function in this direction.

As in the method of Box and Wilson, as well as other methods for searching the extremes of the response function, which are based on gradient method is used not the gradient, and its evaluation. We present a general formulation of the gradient estimation problem and its solution.

Let the response function

$$
\eta=f\left(X_{1}, X_{2}, \ldots, X_{k}\right)
$$

defined in $\mathrm{G} \subset \mathbf{R}^{\mathrm{k}}$. Consider an arbitrary point $\bar{X}^{0} \in G$. Using a point $\bar{X}^{0}=\left(X_{1}^{0}, X_{2}^{0}, \ldots, X_{k}^{0}\right)^{\prime}$ as the center of the plan [4], see full or fractional factorial experiment $[1,2]$. Denoted by $X_{i l}^{0}$ taken by the variable $X_{i}(i=1,2, \ldots, k)$ in the $l$-th level $(l=1,2)$. Upper $X_{i 2}^{0}$ and lower $X_{i 1}^{0}$ levels chosen symmetrically relative to the center plan to satisfy the condition $X_{i}=\left(X_{i 1}^{0}+X_{i 2}^{0}\right) / 2(i=1,2, \ldots, k)$ [3]. It is obvious that the encoded variable

$$
x_{i}=\left(X_{i}-X_{i}^{0}\right) / S_{i}^{0}, i=1,2, \ldots, k,
$$

where $S_{i}^{0}=\left(X_{i 2}^{0}-X_{i 1}^{0}\right) / 2$ - the interval of variation.

Express the response function (1) through coded variables:

$$
\eta=f_{1}\left(x_{1}, x_{2}, \ldots, x_{k}\right)
$$

The transition to the new variables means the transfer origin to the point $\bar{X}^{0}$ and compression (tension) on the coordinate axes [1]. 


\section{The Task of Estimating the Gradient}

The task of estimating the gradient will understand some estimate of the gradient of the response function $f_{1}\left(x_{1}, X_{2}, \ldots, X_{k}\right)$ in point $x^{0}=\left(x_{1}^{0}, X_{2}^{0}, \ldots, X_{k}^{0}\right)^{\prime}$, where $x_{i}^{0}=0 \quad(\mathrm{i}=1, \ldots, \mathrm{k})$. Suppose that in a neighborhood of $\left(x_{1}^{0}, x_{2}^{0}, \ldots, x_{k}^{0}\right)^{\prime}$ function (3) can be decomposed by the Taylor series expansion of the form:

$$
\begin{gathered}
\eta=f_{1}\left(x_{1}, x_{2}, \ldots, x_{k}\right)=f_{1}\left(x_{1}^{0}, x_{2}^{0}, \ldots, x_{k}^{0}\right)+\sum_{i=1}^{k} \frac{\partial f_{1}}{\partial x_{i}^{0}} x_{i}+ \\
+\frac{1}{2} \sum_{i=1}^{k} \sum_{j=1}^{k} \frac{\partial^{2} f_{1}}{\partial x_{i}^{0} \partial x_{j}^{0}} x_{i} x_{j}+o\left(\left\|x-x^{0}\right\|^{2}\right) .
\end{gathered}
$$

Using the values

$$
\begin{gathered}
\beta_{0}^{0}=f_{1}\left(x_{1}^{0}, x_{2}^{0}, \ldots, x_{k}^{0}\right) ; \beta_{i}^{0}=\frac{\partial f_{1}}{\partial x_{i}^{0}} ; \quad \beta_{i i}^{0}=\frac{\partial^{2} f_{1}}{2 \partial\left(x_{i}^{0}\right)^{2}} ; \\
\beta_{i j}^{0}=\frac{\partial^{2} f_{1}}{\partial x_{i}^{0} \partial x_{j}^{0}},
\end{gathered}
$$

we can write the response function

$$
\begin{aligned}
& \eta= \\
& \beta_{0}^{0}+\sum_{1 \leq i \leq k} \beta_{i}^{0} x_{i}+\sum_{1 \leq i<j \leq k} \beta_{i j}^{0} x_{i} x_{j}+\sum_{1 \leq i \leq k} \beta_{i i}^{0} x_{i}^{2}+o\left(\left\|x-x^{0}\right\|^{2}\right) .
\end{aligned}
$$

It should be noted that grad $f_{1}\left(x^{0}\right)=\left(\beta_{1}^{0}, \beta_{2}^{0}, \ldots, \beta_{k}^{0}\right)^{\prime}$. Therefore, the problem of estimating the gradient is reduced to finding the OLS estimates of the unknown parameters $\beta_{1}^{0}, \beta_{2}^{0}, \ldots, \beta_{k}^{0}$. Let $\bar{D}=\left(x_{i u}\right) \quad(i=1,2, \ldots, k ; u=1,2, \ldots, N)$ matrix of full or fractional factorial plan with center $x^{0}=\left(x_{1}^{0}, x_{2}^{0}, \ldots, x_{k}^{0}\right)^{\prime}$. For example, a full factorial plan matrix type $2^{2}[6]$ has the form:

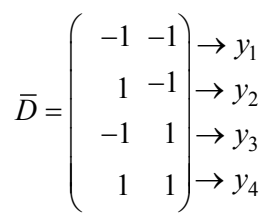

for the case when $\eta=f_{1}\left(x_{1}, x_{2}\right)$. For simplicity we assume that in the region $\mathrm{T}=\left\{\left(x_{1}, x_{2}, \ldots, x_{k}\right)^{\prime}:-1 \leq x_{i} \leq 1\right\}$ response surface quite accurately approximated by a hyperplane, i.e.

$$
\eta \approx \beta_{0}^{0}+\sum_{i=1}^{k} \beta_{i}^{0} X_{i}
$$

Then, if $\mathbf{X}=\left(x_{j u}\right)(j=0,1, \ldots, k ; u=1,2, \ldots, N)$ - matrix of independent variables corresponding to the matrix plan $\bar{D}=\left(x_{i u}\right)$ and the response function (5), The OLS estimator $\beta_{i}^{0}$ is

$$
\hat{\beta}_{j}^{0}=\frac{1}{N} \sum_{u=1}^{N} x_{j u} y_{u}, \quad j=0,1, \ldots, k,
$$

where $y_{1}, y_{2}, \ldots, y_{N}$ - supervision in plan points.

As $\bar{\beta}_{1}^{0}, \bar{\beta}_{2}^{0}, \ldots, \bar{\beta}_{k}^{0}$ are estimates of the components of the gradient $\beta_{1}^{0}, \beta_{2}^{0}, \ldots, \beta_{k}^{0}$ or partial $\frac{\partial f_{1}}{\partial x_{1}^{0}}, \frac{\partial f_{1}}{\partial x_{2}^{0}}, \ldots, \frac{\partial f_{1}}{\partial x_{k}^{0}}$, then the OLS estimate of the gradient of the response function at the point $\left(X_{1}^{0}, X_{2}^{0}, \ldots, X_{k}^{0}\right)^{\prime}$ equal

$$
\operatorname{grad} f_{1}\left(x_{1}^{0}, x_{2}^{0}, \ldots, x_{k}^{0}\right)=\left(\hat{\beta}_{1}, \hat{\beta}_{2}, \ldots, \hat{\beta}_{k}\right)^{\prime}
$$

Evaluation of the gradient of the response ( 6) coincides with the point of the $\left(X_{1}^{0}, X_{2}^{0}, \ldots, x_{k}^{0}\right)^{\prime}$ estimates its gradient at this point. Evaluating the gradient in the problem of finding an extremum is assumed that the response function near of the center plan factorial experiment accurately enough approximated by a hyperplane. In real situations, this condition is often not fulfilled. In this case evaluation of the gradient component may be biased. There is a relationship between the components of the gradient estimates and the estimates of the model parameters used to describe the response surface. Knowing bias of the estimates of the model parameters, we can find the bias of the gradient estimation. This implies an extremely important practical research conclusion: even the use of crude models to describe the response surface can not lead to a shift in the center of the gradient evaluation plan. This fact partly explains the reason for the relatively successful application of the method of Box and Wilson in applied research with a relatively crude approximation of the response surface.

Problem of unbiased estimation of the gradient gr $\operatorname{adf}\left(x_{1}^{0}, x_{2}^{0}, \ldots, x_{k}^{0}\right)$ during the decomposition of the response function $\eta=f\left(x_{1}^{0}, x_{2}^{0}, \ldots, x_{k}^{0}\right)$ of the Taylor series (4) near of the center plan [6] $x^{0}=\left(x_{1}^{0}, x_{2}^{0}, \ldots, x_{k}^{0}\right)^{\prime}$ is to find unbiased estimates of the components of the gradient $\beta_{i}=\frac{\partial f}{\partial x_{i}^{0}}$, where $x_{i}^{0}=0 \quad(i=1,2, \ldots, k)$.

Consider the case where

$$
\eta=f\left(x_{1}, x_{2}, \ldots, x_{k}\right)=\beta_{0}+\sum_{1 \leq i \leq k} \beta_{i} x_{i}+\sum_{1 \leq i<j \leq k} \beta_{i j} x_{i} x_{j}+\sum_{i \leq i \leq k} \beta_{i i} x_{i}^{2}
$$

Obviously, $\operatorname{gradf}\left(x_{1}^{0}, x_{2}^{0}, \ldots, x_{k}^{0}\right)=\left(\beta_{1}, \beta_{2}, \ldots, \beta_{k}\right)^{\prime}$. The following lemma is fair:

Lemma 1. For the response function of the form (7) using a fractional factorial design type $2^{\mathrm{k}-\mathrm{q}}$ OLS-estimate of the gradient $\operatorname{gr} a d f\left(x_{1}^{0}, x_{2}^{0}, \ldots, x_{k}^{0}\right)$ will be unbiased if and only if the variables $x_{1}, x_{2}, \ldots, x_{k}$ satisfy 


$$
\begin{gathered}
\pm \bar{X}_{i} \neq \bar{x}_{j}, \quad i, \quad j=1,2, \ldots, k, \quad i \neq j \\
\pm \bar{X}_{i} \neq \bar{x}_{I} \otimes \bar{x}_{s}, \quad i, \quad l, \quad s=1,2, \ldots, k, \quad l \neq s
\end{gathered}
$$

Proof: Let $\bar{D}=\left(\bar{x}_{i}\right) \quad(i=1,2, \ldots, k)-$ matrix factor type $2^{\mathrm{k}-\mathrm{q}}$ and also conditions (8), (9) are satisfied. Write response function (7) as

$$
\eta=\sum_{I \leq i \leq k} \beta_{i} X_{i}+\beta_{0}+\sum_{1 \leq i<j \leq k} \beta_{i j} X_{i} X_{j}+\sum_{I \leq i \leq k} \beta_{i i} X_{i}^{2}
$$

Then not hard to believe that the columns of the matrix of

independent variables

$$
X=\left(\widetilde{X}_{j}\right) \quad(j=1,2, \ldots, p+1)
$$

corresponding response function (10), satisfy the conditions of

$$
\begin{gathered}
\left\|\widetilde{\boldsymbol{X}}_{j}\right\|^{2}=N, \quad j=1,2, \ldots, p+1 ; \\
\widetilde{\boldsymbol{X}}_{i}^{\prime} \widetilde{\boldsymbol{X}}_{j}=0, \quad i=1,2, \ldots, k, \quad j=1,2, \ldots, p+1 ; \quad i \neq j
\end{gathered}
$$

It follows that the matrix $X$ can be represented as a matrix block [1]

$X=\left(X^{\circ}, X^{*}\right) \quad, \quad$ where $\quad X^{0}=\left(\tilde{x}_{j}\right)(j=1,2, \ldots, \kappa)$, $X^{*}=X^{o} R$. For two arbitrary plans $\xi_{1}\left(N_{0}\right)$ and $\xi_{2}\left(N_{0}\right)$ with matrix $\bar{D}_{1}^{*}=\left(x_{i u}^{(1)}\right)$ and $\bar{D}_{2}^{*}=\left(x_{i u}^{(2)}\right)$ $\left(\mathrm{i}=1,2 \ldots, \mathrm{k} ; \mathrm{u}=1, \ldots, \mathrm{N}_{0}\right)$ there is orthogonal matrix $\mathrm{R}$ : $\bar{D}_{1}^{*}=R \bar{D}_{2}^{*}$, whence $R=\bar{D}_{1}^{*}\left(\bar{D}_{2}^{*}\right)^{-1} \quad$ [1]. According (12) $X^{0^{\prime}} X^{*}=0$ and hence $[1,2]$, OLS - evaluation $\hat{\beta}^{\circ}=\left(\hat{\beta}_{1}, \hat{\beta}_{2}, \ldots, \hat{\beta}_{h}\right)^{\prime}$ gradient vector $\left(\beta_{1}, \beta_{2}, \ldots, \beta_{h}\right)^{\prime}$ gradient vector and equal $\hat{\beta}_{j}=\left(X^{0^{\prime}} X\right)^{-1} X^{0^{\prime}} Y$ where $Y=\left(y_{1}, y_{2}, \ldots, y_{N}\right)^{\prime}$ - vector of supervision.

Because the columns of the matrix $X^{0}$ are pair wise orthogonal [1], then

$$
\hat{\beta}_{j}=(N)^{-1} \tilde{x}_{j} Y, \quad j=1, \ldots, \kappa
$$

It is easy to see that if the OLS - estimate of the gradient $\operatorname{gradf}\left(x_{1}^{0}, x_{2}^{0}, \ldots, x_{k}^{0}\right)$ is unbiased, then (11), (12).

Consequence1. If the matrix of the plan $\bar{D}=\left(x_{i u}\right)(i=1, \ldots, k ; u=1, \ldots, N)$ is a matrix of the full plan $2^{\mathrm{k}}$, then conditions (11), (12) is carried out for any $k$ and therefore unbiased OLS - estimate of the gradient exists and is defined by (13).

Consequence2. If the conditions (11) and (12) of Lemma 1 are satisfied, then for the approximation of the response surface can be used dependence of the form

$$
\eta=\beta_{0}+\beta_{1} x_{1}+\ldots+\beta_{k} x_{k}
$$

In this case, OLS - estimate of the gradient models (7) and (13) is the same.

Note that condition (11), (12) is performed only in the case where the fractional resolutio replicates $2^{\mathrm{k}-\mathrm{q}}$ greater than three [2]. Therefore reformulate Lemma 1 with that in mind. Lemma 2. Application of fractional replicate type $2^{\mathrm{k}-\mathrm{q}}$ allows to obtain an unbiased OLS - estimate of the gradient $\operatorname{gradf}\left(x_{1}^{0}, x_{2}^{0}, \ldots, x_{k}^{0}\right)$ of the response function (7) at the point $\left(x_{1}^{0}, x_{2}^{0}, \ldots, x_{k}^{0}\right)^{\prime}$ if and only if its resolution is greater than three..

\section{Examples of Specific Implementations}

Example 1. Response function $\eta=f\left(x_{1}, x_{2}, x_{3}, x_{4}\right)$ has the form (7). It is easy to see that half-replicate $2_{I V}^{4-1}$ with defining contrast $l=x_{1} x_{2} x_{3} x_{4}$ as a resolution equal to four, and by Lemma 2 allows to obtain an unbiased estimate of the gradient $\operatorname{gradf}(0,0,0,0)$ of the response function in the center of the plan. Obvious then

$$
\operatorname{grad} f(0,0,0,0)=\left(\hat{\beta}_{1}, \hat{\beta}_{2}, \hat{\beta}_{3}, \hat{\beta}_{4}\right)^{\prime}
$$

where

$$
\begin{aligned}
& \hat{\beta_{1}}=\frac{1}{4}\left(-y_{1}+y_{2}-y_{3}+y_{4}-y_{5}+y_{6}-y_{7}+y_{8}\right) ; \\
& \hat{\beta}_{2}=\frac{1}{4}\left(-y_{1}-y_{2}+y_{3}+y_{4}-y_{5}-y_{6}+y_{7}+y_{8}\right) ; \\
& \hat{\beta_{3}}=\frac{1}{4}\left(-y_{1}-y_{2}-y_{3}-y_{4}+y_{5}+y_{6}+y_{7}+y_{8}\right) ; \\
& \hat{\beta}_{4}=\frac{1}{4}\left(-y_{1}+y_{2}+y_{3}-y_{4}+y_{5}-y_{6}-y_{7}+y_{8}\right) .
\end{aligned}
$$

If for this purpose use half-replicate $2_{I I I}^{4-1}$ with defining contrast $l=x_{1} x_{2} x_{4}$, then the OLS-estimate of the gradient will be biased, and [2]

$$
\begin{gathered}
M \operatorname{grad} f(0,0,0,0)= \\
\left(M\left\{\hat{\beta}_{1}\right\}, M\left\{\hat{\beta}_{2}\right\}, M\left\{\hat{\beta}_{3}\right\}, M\left\{\hat{\beta}_{4}\right\}\right)^{\prime}= \\
=\left(\beta_{1}+\beta_{24}, \beta_{2}+\beta_{14}, \beta_{3}, \beta_{4}+\beta_{12}\right)^{\prime} .
\end{gathered}
$$

In this case, only an estimate of the gradient component $\beta_{3}$ is biased; estimates other components are biased.

Example 2. Response function $\eta=f\left(x_{1}, x_{2}, \ldots, x_{7}\right)$ has the form (3). Let's make sure that the $1 / 4$ - replicate $2^{7-2}$, defined 
by generating relations $x_{6}=x_{1} x_{2} x_{3}, x_{7}=x_{3} x_{4} x_{5}$, as a resolution equal to four [1], and therefore allows to obtain an unbiased OLS-estimate of the gradient in the center of the plan. Indeed, determining contrasts defining fractional replicate $[2,5]$ are equal $1=x_{1} x_{2} x_{3} x_{6} ; 1=x_{3} x_{4} x_{5} x_{7}$.

Out of here defining the generalized contrast [6] $1=x_{1} x_{2} x_{3} x_{6}=x_{3} x_{4} x_{5} x_{7}=x_{1} x_{2} x_{3} x_{5} x_{6} x_{7} \quad$ has $\quad$ a resolution equal to four, i.e., fractional replicate is a replicate of $2_{I V}^{7-2}[1]$. It is easy to check that the class of fractional replicate $2^{7-3}$ fractional replicate does not exist, allowing for unbiased estimation of the gradient [1,2, and 6].

These examples illustrate a general approach to solving the problem of estimating the gradient in situations where the model approximately describes the response surface [3-5]. From this discussion it follows that in some important practice $[3,4]$ cases is possible to match gradient estimates for models of varying complexity. Is a very complex problem of comparing the effectiveness of plans in estimating the gradient.

2. Assume that the distribution of the directions grad $f(\bar{x})=\left(\beta_{1}^{0}, \beta_{2}^{0}, \ldots, \beta_{k}^{0}\right)^{\prime}$ know that it belongs to some parameter family of distributions. In case of continuous random variable it means that known views density $p$ (grad $f(\bar{x}) / \theta$ ), but not know the value of the parameter $\mathrm{K}$ determining the concrete density [5,7]. Parameter $\theta$ can be a vector. For example, for a normal distribution $\theta=(\mu, \sigma)$

$$
p(\operatorname{grad} f(\bar{x}) / \theta)=\frac{1}{\sigma \sqrt{2 \pi}} \exp \left\{-(\operatorname{grad} f(\bar{x})-\mu)^{2} / 2 \sigma^{2}\right\} .
$$

In the case of a discrete random variable $p(\operatorname{grad} f(\bar{x}) / \theta)$, will denote the probability $p(X=\operatorname{grad} f(x))$. For example, for Poisson distribution

$$
p(\operatorname{grad} f(\bar{x}) / \theta)=\frac{\theta^{x}}{x !} e^{-\theta}, x=0,1,2,3, \ldots
$$

Let $\tau(\theta)$ be a numeric parameter of interest to us. For example, $\tau(\theta)=\theta, \tau(\mu, \sigma)=\mu, \tau(\mu, \sigma)=\mu / \sigma$.

Consider the problem of estimation of the gradient, consisting in the construction of a function $\operatorname{gr} \hat{a} d f(\bar{x})$ $=\left(x_{1}, \ldots, x_{n}\right)$, to the substitution of the instead of the arguments $x_{1}, \ldots, x_{n}$ sampling data of the directions of the gradient we got the numbers, "close" to $\tau(\theta)$. This proximity can only be done in average. Therefore, the requirements to the quality of the estimates of the gradient, is formulated in probabilistic terms relating to the distribution of the assessments covered as random values $[7,8]$. This is the requirement to estimate values in most of the experiments were close to the estimated value of the parameter can be formulated as the following definition.

Definition 1. Estimate grad $f(\bar{x})=\left(\mathrm{x}_{1}, \ldots, \mathrm{x}_{\mathrm{n}}\right)$ is called for the unbiased $\tau(\theta)$, if $M_{\theta} \operatorname{gradf}(\bar{x})=\tau(\theta)$ for all $\theta$, where $\mathrm{M}_{\theta}$ symbol mathematical expectation, provided that the random vector $\operatorname{grad} f(\bar{x})=\left(x_{1}, \ldots, x_{n}\right)$ have the distribution

$$
L(\operatorname{grad} f(\bar{x}) / \theta)=p\left(\operatorname{grad} f\left(x_{1}\right) / \theta\right) \ldots p\left(\operatorname{grad} f\left(x_{n}\right) / \theta\right) .
$$

But, as a rule, only one unbiasedness requirement does not emit estimation for unambiguously. Therefore, the following desirable requirement is the requirement of minimum of variance of this estimation [7]. To statistics might serve as a good estimate of this parameter $\tau(\theta)$, it is necessary that the distribution of this statistics was concentrated in sufficiently close to the unknown value $\tau(\theta)$, so that the probability of large deviations of the statistics from $\tau(\theta)$ was quite small [7]. Then the systematic application of repeated this statistics as an estimation of the characteristics on the average will be obtained sufficient accuracy. The probability of large deviations will be small, and they will be rare.

Thus, among all unbiased estimates $\operatorname{gradf}(\bar{x})$ for $\tau(\theta)$ more desirable is the estimation of the gradient, which has a minimum variance

$$
D_{\theta}(\operatorname{gradf}(\bar{x}))=M(\operatorname{grad} f(\bar{x})-\tau(\theta))^{2} \text { for all } \theta .
$$

Definition The unbiased estimate of the gradient with minimum variance is estimated $\operatorname{grad}^{*} f(\bar{x})$ that $D\left(\operatorname{grad}^{*} f(\bar{x})\right) \leq D(\operatorname{grad} f(\bar{x}))$ for all $\theta$ for any unbiased estimate the gradient $\operatorname{grad} f(\bar{x})$ parameter $\tau(\theta)$.

These requirements generally provide the estimate clearly, if such an estimate gradient exists at all. The existence of the unbiased estimate of the gradient with minimum variance (UEGMV) takes place, not always, as far as the variance for these estimates should be minimal evenly $\theta$. This fact is perhaps the most serious argument against such strong requirements such as unlimited increasing the number of observations, i.e. $\lim _{n \rightarrow \infty} P\{|\hat{\operatorname{gra} d f}(\bar{x})-\tau(\theta)|<\varepsilon\}=1 \quad$ for $\forall \varepsilon>0$

So, the viability of the gradient estimation means that if a sufficiently large number of observations $n$ with arbitrarily high accuracy deviation estimate the gradient from the true parameter value less any pre-specified value.

To unbiased estimate of the gradient was valid enough to variance estimation tends to zero if $\mathrm{n} \rightarrow \infty$ (this follows from the Chebyshev inequality[7]).

Example 3. Let's show that the estimates of the gradient

$$
\begin{aligned}
& \text { a. } \bar{x}=\frac{1}{n} \sum_{1}^{n} \operatorname{gradf}\left(x_{i}\right) \text { - the sample mean, } \\
& \text { b. } \quad \bar{S}^{2}=\frac{1}{n} \sum_{1}^{n}\left(\operatorname{gra} d f\left(x_{i}\right)-\bar{x}\right)^{2} \text { - sample variance, }
\end{aligned}
$$

Are valid estimates.

Decision. According to the law of large numbers 


$$
\begin{aligned}
& P\left\{\left|\frac{\sum_{1}^{n} \operatorname{gradf}\left(x_{i}\right)}{n}-M(\operatorname{gradf}(\bar{x}))\right|<\varepsilon\right\} \rightarrow 1, n \rightarrow \infty \\
& \bar{x}=\frac{1}{n} \sum_{1}^{n} \operatorname{gradf}\left(x_{i}\right)
\end{aligned}
$$

b)

$$
\begin{aligned}
& \bar{S}^{2}=\frac{1}{n} \sum_{1}^{n}\left(\left(\operatorname{grad} f\left(x_{i}\right)\right)^{2}-2 \operatorname{gradf}\left(x_{i}\right) \bar{x}+\bar{x}^{2}\right)= \\
& =\frac{1}{n}\left(\sum_{1 i}^{n}\left(\operatorname{gradf}\left(x_{i}\right)\right)^{2}-2 \bar{x} \sum \operatorname{grad} f\left(x_{i}\right)+n \bar{x}^{2}\right)= \\
& =\frac{\sum\left(\operatorname{grad} f\left(x_{i}\right)\right)^{2}}{n}-2 \bar{x}^{2}+\bar{x}^{2}=\frac{\sum\left(\operatorname{gradf}\left(x_{i}\right)\right)^{2}}{n}-\bar{x}^{2}
\end{aligned}
$$

the first member is the arithmetic mean of the random variables $\left(\operatorname{gradf}\left(x_{i}\right)\right)^{2}$ hence it converges in probability to $M\left(\operatorname{gradf}\left(x_{i}\right)\right)^{2}$, the second term converges in probability $M\left(\operatorname{gradf}\left(x_{i}\right)\right)^{2}$. This means that $\bar{S}^{2}$ the probability converges to a $M\left(\operatorname{gradf}\left(x_{i}\right)\right)^{2}-\left(M\left(\operatorname{gradf}\left(x_{i}\right)\right)\right)^{2}$ $=D\left(\operatorname{gradf}\left(x_{i}\right)\right)$. Hence, the estimate is valid, but it is not unbiased.

Example 4. (The existence of several unbiased estimates of the gradient.)

gr $\hat{a} d f\left(x_{1}\right), \ldots, \quad \hat{g r} \hat{a} d f\left(x_{n}\right)$ - independent identically distributed random variables having a Poisson distribution $P_{\theta}\left\{\operatorname{gradf}\left(x_{i}\right)=k\right\}=\frac{\theta^{k}}{k !} e^{-\theta}$.

Estimate $\quad \bar{S}_{1}^{2}=\frac{1}{n-1} \sum\left(\operatorname{gradf}\left(x_{i}\right)-\operatorname{gr} \hat{a} d f(x)\right)^{2} \quad$ is the unbiased estimate for the variance. The fact that the Poisson distribution the mean and the variance $\theta$ and grad $f\left(x_{1}\right)$ is the unbiased estimate of $\theta$ hence, that $\operatorname{gradf}\left(x_{1}\right)$ is the unbaised estimate for variance $\theta$. In addition, any combination $\alpha g r a d f(x)+(1-\alpha) S_{1}^{2}$ is the unbiased estimate

$$
\begin{gathered}
\theta(\alpha \in(-\infty, \infty)) \text { i.e. } \\
M\left(\alpha g r \vec{a} d f(x)+(1-\alpha) S_{1}^{2}\right)= \\
=\alpha M(\operatorname{gr} a d f(x))+(1-\alpha) M S_{1}^{2}= \\
=\alpha \theta+(1-\alpha) \theta=0 .
\end{gathered}
$$

However, estimate $\operatorname{gr} a d f\left(x_{1}\right)$ has the lowest variance.
Example 5. (When there are no unbiased assessment of the gradient.)

One observation $\operatorname{gr} \hat{a} d f(\bar{x})$, where $\hat{\operatorname{gr}} \hat{a} d f(\bar{x})$ is a random variable which has a Poisson distribution with an unknown parameter $\theta$

$$
p_{\theta}(\operatorname{grad} f(x)=k)=\frac{\theta^{k}}{k !} e^{-\theta}, k=0,1, \ldots
$$

Let's show that there is not unbiased estimate the gradient. Decision. Suppose that $\hat{\operatorname{gr}} \hat{a} d f(\bar{x})$ is the unbiased estimate $\tau(\theta)$ i.e. $M_{\theta} \operatorname{grad} f(x)=\tau(\theta)$ whence it follows that

$$
\sum_{k=0}^{\infty} \frac{\hat{g r a d f}(x) e^{-\theta} \theta^{k}}{k !}=\frac{1}{\theta}
$$

Whence it follows that

$$
\sum_{k=0}^{\infty} \frac{\operatorname{gr} \hat{a} d f(x)}{k !} \theta^{k}=\frac{e^{\theta}}{\theta}=\frac{1}{\theta} \sum_{k=0}^{\infty} \frac{\theta^{k}}{k !} .
$$

Finally $\sum \frac{\operatorname{grad} f(x)}{k !} \theta^{k}=\sum_{k=-1}^{\infty} \frac{1}{(k+1) !} \theta^{k}$.

And as easy to understand, there is no function

$\operatorname{gr} \operatorname{adf}(x)$

[7], for which the last equality it would be fair for all $\theta>0$.

Example 6. (when unbiased estimate of the gradient is absurd)

A random variable $\operatorname{grad} f(x)$ has a geometric distribution [8]:

$$
p\{\operatorname{grad} f(x)=k\}=q^{k} p, k \geq 0, p+q=1
$$

(the number of failures preceding the first success in a sequence of Bernoulli trials).

The unbiased estimate of the parameter $p$ is the estimate of

$$
\hat{\operatorname{grad} f}(x)= \begin{cases}1, & x=0, \\ 0, & x>0 .\end{cases}
$$

Indeed,

$$
\begin{aligned}
\sum_{k=0}^{\infty} \hat{\operatorname{gradf}}(k) q^{k} p=p, & \sum_{k=0}^{\infty} \hat{\operatorname{gradf}}(x) q^{k}=1 \Rightarrow \\
\hat{\operatorname{gr} \hat{a} d}(k) & = \begin{cases}1, & k=0, \\
0, & k>0 .\end{cases}
\end{aligned}
$$

However, this estimate of the gradient is absurd, because, for example, if the first success was obtained on the second experiment, i.e. $x=1$, it is absurd to say that the probability of success $p$ is equal to 0 .

Let's prove that UEGMV only in the sense that if $\hat{\operatorname{grad}} f_{1}(x)$ and $\hat{\operatorname{grad}} f_{2}(x)$ there are two UEGMV for $\tau(\theta)$, then $\operatorname{grad}_{1}(x)=\hat{\operatorname{grad}} f_{2}(x)$ with probability one. 
For that, make $\operatorname{gradf}(x)=\mathrm{t}_{1}, \operatorname{gradf}_{2}(x)=\mathrm{t}_{2}$ and

$$
\operatorname{gradf} f_{3}(x)_{t_{3}} \text {. }
$$

In fact, consider a new estimate $t_{3}=\frac{1}{2}\left(t_{1}+t_{2}\right)$, for which

$$
\begin{aligned}
& M_{\theta} t_{3}=\frac{1}{2}\left(M t_{1}+M t_{2}\right)=\tau(\theta), \\
& D t_{3}=\frac{1}{4}\left(D t_{1}+D t_{2}+2 \operatorname{cov}\left(t_{1}, t_{2}\right)\right) .
\end{aligned}
$$

It is known that $\operatorname{cov}\left(t_{1}, t_{2}\right) \leq{\sqrt{D t_{1} D t}}_{2}[7,9,10]$, and the sign of equality occurs only when almost everywhere

$$
\left(\mathrm{t}_{1}-\tau\right)=\mathrm{k}(\theta)\left(\mathrm{t}_{2}-\tau\right)
$$

Where $\mathrm{k}(\theta)$ any function from $\theta$. If we mark $\mathrm{Dt}_{1}=\mathrm{Dt}_{2}=\mathrm{V}$, we have shown that $\operatorname{cov}\left(\mathrm{t}_{1}, \mathrm{t}_{2}\right) \leq \mathrm{V}$, and therefore $\mathrm{Dt}_{3} \leq \mathrm{V}$, with the sign of equality occurs only when the condition (14).

Find $\mathrm{k}(\theta)$. Know $V=\operatorname{cov}\left(t_{1}, t_{2}\right)=k(\theta) V$ whence it follows that $\mathrm{k}(\theta)=1$ and therefore $\mathrm{t}_{1}=\mathrm{t}_{2}$ and correspondingly $\operatorname{gradf_{1}(x)=} \operatorname{gradf}_{2}(x)$ almost everywhere.

These examples allow you to move on to more complex tasks [11], namely to the problems of comparison of the effectiveness of plans at the estimation of the gradient.

\section{Conclusions}

Thus, the existence of the unbiased estimate of the gradient with minimum variance occurs not always, as far as the variance for these estimates should be minimal uniformly in the parameter. The viability of the gradient estimation means that if a sufficiently large number of observations $n$ with arbitrarily high accuracy deviation estimate the gradient from the true parameter value less any pre-specified value. To unbiased estimate of the gradient was wealthy enough to variance estimation tends to zero $\mathrm{n} \rightarrow \infty$.

\section{REFERENCES}

[1] Asaturyan V.I. Experimental design theory. -M., Radio and communication, 1983.

[2] Nalimov V.V. The theory of experiment. _M., Science, 1971. $-208 p$.

[3] Experimental design theory mathematical modeling of the heat transfer rate/Sidnyaev N.I., Levin V.A., Afonina N.E., Kats A.M.//Engineering-physical magazine. -2002. -T.75, №2 -p.132-138.

[4] Sidnyaev N.I. Research of formation liquid metal media in the conditions of the surface mass transfer methods of the theory of planning of experiment/Izvestia of higher schools. Ser. Mechanical engineering. 2002. № 5. p. 11-20.

[5] Sidnyaev N.I. Experimental design theory and static data analysis: M: yurayt Publishing house, 2011.

[6] Sidnyaev N.I. Introduction to the theory of planning of experiment. Tutorial/ N.I. Sidnyaev, N.T. Vilisova -M: Izd-vo MGTU im. N. E. Bauman, 2011.

[7] Borodin A.N. An elementary course in probability theory and mathematical statistics - Spb, Lan, 1999.

[8] Sidnyaev N.I. Probability theory and mathematical statistics: a manual. -M.: Izd-vo «Yurait», 2011.

[9] Abdenov A.J., Avdeenko T.V., Denisov V.I. Mathematical methods of analysis and design of experiment. Analysis, optimal filtering and disign of experiment in dynamic systems: Method. Design. -Novosibirsk: publishing house of NGTU, 1995. -Part 2. - 59p.

[10] Jonson I., Lion F. Statistics and experimental design in science and engineering. -M. : Mir, 1981. -516 p

[11] Slotin U.S. Compositional design of regression experiment. M.: Knowlegem 1983. 